5. Dedoussis GV, Kapiri A, Samara A, et al. Expression of inflammatory molecules and associations with BMI in children. Eur J Clin Invest 2010;40(5):388-92.

6. Alberti KG, Zimmet $P$, Shaw J, et al. The metabolic syndrome--a new worldwide definition. Lancet 2005;366(9491):1059-62.

7. Vallianou NG, Evangelopoulos AA, Panagiotakos DB, Georgiou AT, et al. Associations of acute-phase reactants with metabolic syndrome in middle-aged overweight or obese people. Med Sci Monit 2010;16(2):CR56-60.

8. Zimmermann E, Anty R, Tordjman J, et al. C-reactive protein levels in relation to various features of non- alcoholic fatty liver disease among obese patients. J Hepatol 2011;55(3):660-5.

9. Bril F, Ortiz-Lopez C, Lomonaco R, et al. Clinical value of liver ultrasound for the diagnosis of nonalcoholic fatty liver disease in overweight and obese patients. Liver Int 2015;35(9):2139-46.

10. BogdańskiP, ChyrekR,Pupek-MusialikD, etal. Evaluation of selected acute phase proteins in patients with metabolic syndrome. Pol Merkur Lekarski 2006;21(121):12-4.

11. ParkSH, Kim CH, Kim DJ, etal.Diagnostic value of multiple biomarker panel for prediction of significant fibrosis in chronic hepatitis C. Clin Biochem 2011;44(17-18):1396-9.

\title{
Caracterización de la consulta por intoxicación medicamentosa en los adolescentes en el Hospital Municipal de Bahía Blanca, Buenos Aires
}

\author{
Characterization of drug poisoning among adolescents seen at the municipal \\ hospital of Bahía Blanca, Province of Buenos Aires, Argentina
}

\author{
Dr. Ignacio Buffone $e^{a}$ Dra. Mabel Dejter ${ }^{a}$, Dra. Eliana Fortunatti ${ }^{a}$, Dra. Florencia García Elliot ${ }^{a}$, \\ Dra. Camila Irazabal ${ }^{a}$ Dr. Rubén Marlia ${ }^{a}$ Dra. Daiana Mujica ${ }^{a}$, Dra. Marianela Parroua, \\ Dra. Mariana Romano ${ }^{a}$ Dra. Gina Speciale ${ }^{a}$ y Dra. Agostina Werneke ${ }^{a}$
}

\section{RESUMEN}

Introducción. La consulta por intoxicación medicamentosa en los niños se da con frecuencia en los servicios de emergencias médicas; en la adolescencia, es cuando ocurren de forma intencional.

Objetivo. Caracterizar los patrones epidemiológicos de la intoxicación medicamentosa en adolescentes ingresados al Hospital Municipal de Bahía Blanca.

Material y método. Estudio descriptivo, basado en la revisión dehistorias clínicas de pacientes de 10 a 19 años que consultaron porintoxicación medicamentosa, entre los años 2012 y 2016. Las variables analizadas fueron edad, sexo, fármaco consumido, causa, manifestaciones clínicas y días de internación.

Resultados. Se recopilaron 72 pacientes. La edad media fue de 16 años, razón mujer-hombre de 2,5:1. Predominaron las intoxicaciones por ansiolíticos $(30 \%)$, seguidas de las

a. Servicio de Clínica Pediátrica, Hospital Municipal de Agudos “Dr. Leónidas Lucero”, Bahía Blanca,

provincia de Buenos Aires.

Correspondencia:

Dr. Ignacio Buffone: nacho_b89@hotmail.com

Financiamiento: Ninguno.

Conflicto de intereses: Ninguno que declarar.

Recibido: 16-7-2017

Aceptado: 21-12-2017 polimedicamentosas (25\%). El 95\% manifestaron intención suicida, de los cuales el 55\% ya tenían antecedentes.

Conclusión. La intoxicación medicamentosa ocurrió, predominantemente, en mujeres, y se destacó el gran porcentaje de adolescentes que presentaba episodios previos.

Palabras clave: adolescente, toxicidad de medicamentos.

http:/ / dx.doi.org/10.5546/aap.2018.279

Texto completo en inglés:

http: / / dx.doi.org/10.5546/ aap.2018.eng.279

Cómo citar: Buffone I, Dejter M, Fortunatti E, et al. Caracterización de la consulta por intoxicación medicamentosa en los adolescentes en el Hospital Municipal de Bahía Blanca, Buenos Aires. Arch Argent Pediatr 2018;116(4):279-282.

\section{INTRODUCCIÓN}

La intoxicación medicamentosa en los adolescentes se ha transformado, en los últimos años, en un serio problema de salud pública, especialmente, en el área pediátrica, dado que son causantes de hasta un $10 \%$ de todos los ingresos a la Unidad de Cuidados Intensivos, con tasas de mortalidad muy variables en los diferentes escenarios de atención. ${ }^{1}$

El espectro de sustancias implicadas en la intoxicación es amplio: los antidepresivos, los ansiolíticos y los antiinflamatorios no 
esteroideos son los grupos farmacológicos de mayor frecuencia en la consulta. ${ }^{2}$ Los intentos de autoeliminación son la principal causa y uno de los problemas más preocupantes que acarrean los niños de este grupo etario. ${ }^{3}$

En la actualidad, las intoxicaciones no son un fenómeno aislado, sino algo cotidiano, sobre todo, en los adolescentes que, en función de los cambios propios de esta etapa evolutiva y por el efecto de un debilitamiento cada vez mayor de los lazos sociales, ven incrementadas las probabilidades de tomar conductas de riesgo.

Visto el importante rol que ocupa este tipo de consultas en los servicios de emergencias y ante la necesidad de obtener datos que complementen las estadísticas locales, es que se decidió realizar un estudio de investigación, con el objetivo de caracterizar los patrones epidemiológicos de la intoxicación medicamentosa ocurrida en adolescentes de 10 a 19 años, ingresados al Servicio de Emergencias del Hospital Municipal de Agudos Leónidas Lucero (HMALL).

\section{METODOLOGÍA}

Estudio descriptivo, retrospectivo, basado en la información recopilada de la revisión de historias clínicas de pacientes de 10 a 19 años que consultaron al Servicio de Emergencias del HMALL por intoxicación medicamentosa, entre el $1^{\circ}$ de enero de 2012 y el 31 de diciembre de 2016. Este período fue dividido en 5 grupos anuales a fin de evaluar también la tendencia de consultas a lo largo del tiempo.

Cabe mencionar que el HMALL de Bahía Blanca es un hospital general de agudos que cuenta con un Servicio de Emergencias que funciona las $24 \mathrm{~h}$. Dispone de una Sala de Internación Pediátrica con 23 camas, donde se internan pacientes con patología de mediana complejidad. Los adolescentes que ingresan en situaciones de vulnerabilidad son evaluados conjuntamente con el psicólogo de guardia: se determina el riesgo y se planifica el abordaje en la emergencia. Si el paciente se interna en la Sala, interviene el equipo interdisciplinario conformado por los hebiatras y los profesionales de la salud mental, con el objetivo de constituir un marco de contención y una red de apoyo que faciliten el seguimiento ambulatorio una vez que sea posible otorgarle el alta hospitalaria.

Los números de historia clínica de cada paciente fueron buscados a través del sistema digital de archivos del Hospital. Parte de los autores extrajeron y volcaron los datos de interés en las planillas realizadas para este fin.

El protocolo de esta investigación fue aprobado por el Comité de Bioética del Hospital, que permitió el acceso a las historias clínicas. En la planilla de recolección de datos, no se colocaron los nombres de los pacientes para respetar su privacidad.

Las variables analizadas fueron edad, sexo, tipo de fármaco consumido, intención, antecedente de intento de autoeliminación, manifestaciones clínicas al ingreso, necesidad de hospitalización, días totales de internación, número de ingresos por año y motivo que había llevado a realizar el evento. Las variables cuantitativas se expresaron como medidas de tendencia central (media, mediana, moda) y de dispersión (rango), y las cualitativas, como porcentajes. Para establecer la significancia al comparar algunas variables, se utilizaron la prueba de chi cuadrado y el coeficiente de correlación, tomando una p menor de 0,05 , con un intervalo de confianza (IC) del 95\%. Para el análisis de datos, se utilizó el software SPSS versión 17.0.

\section{RESULTADOS}

Ochenta pacientes con diagnóstico de intoxicación medicamentosa ingresaron al Servicio de Emergencias durante el período del estudio. Del total, fueron excluidos 8 debido a errores en la codificación diagnóstica, por lo que quedó un total de 72 para el análisis.

La mediana de edad fue de 16 años, con un rango de entre 10 y 19 años. La media y la moda también fueron de 16.

En cuanto a la distribución porcentual de pacientes según el sexo, el $72 \%$ correspondió al femenino (n: 52). La razón mujer-hombre fue de 2,5:1.

En la Tabla 1, se expresa la distribución de la frecuencia de intoxicaciones medicamentosas en los 5 períodos analizados. Se evidencia un importante aumento de la consulta en el año 2015, y se mantienen cifras similares en el resto de los años evaluados. Las diferencias no fueron estadísticamente significativas ( $\mathrm{p}=0,1$; IC 95\%: $0,075-0,42$ ).

Tabla 1. Distribución de frecuencias según los periodos

\begin{tabular}{lccc}
\multirow{2}{*}{ Período } & \multicolumn{3}{c}{ Consultas según el sexo } \\
& Femenino (n) & Masculino (n) & Total (n\%) \\
\hline 2012 & 8 & 5 & $13(18 \%)$ \\
2013 & 5 & 6 & $11(15 \%)$ \\
2014 & 14 & 1 & $15(21 \%)$ \\
2015 & 17 & 5 & $22(31 \%)$ \\
2016 & 8 & 3 & $11(15 \%)$ \\
\hline
\end{tabular}


Con respecto a la frecuencia de consumo según los grupos farmacológicos, los ansiolíticos se encontraron en primer lugar y fueron consumidos por 22 de los 72 pacientes, mientras que la ingesta de polifármacos ocurrió en 18 de ellos (Tabla 2).

En relación con la presentación clínica al momento de la consulta, poco más de la mitad (n: 39) se encontraban asintomáticos, mientras que 24 manifestaron síntomas neurológicos (letargia, mareos, depresión del sensorio, tendencia al sueño); 7 , gastrointestinales (vómitos, dolor abdominal), y solo 2 pacientes presentaron síntomas cardiovasculares (taquicardia, hipotensión).

Del total de los pacientes, el 95\% (n: 68) refirieron intención suicida. El 55\% de ellos tenían antecedentes de tentativas de autoeliminación. Al compararlo por grupo etario, se pudo observar que los adolescentes mayores de 15 años tenían mayor porcentaje de intento de autoeliminación como causa de intoxicación ( $\mathrm{p}=0,037$; IC 95\%: 0,034-0, 041) y mayor proporción de antecedentes $(p=0,034 ;$ IC 95\%: 0,032-0,04). No hubo diferencias significativas al comparar por sexo $(\mathrm{p}=0,798$; IC 95\%: 0,798-0,806).

El conflicto familiar fue el motivo de mayor importancia a la hora de justificar el acto (49\%). Otros motivos encontrados fueron conflicto escolar $(10 \%)$ y conflictos de pareja $(9 \%)$.

De acuerdo con los resultados obtenidos, se observó que el $42 \%$ de los pacientes (N: 30$)$ requirieron internación en la Sala de Clínica Pediátrica o Clínica Médica, dependiendo de la edad del paciente, y el tiempo de estadía promedio fue de 2,5 días, sin diferencias significativas al compararlo por sexo $(p=0,054$; IC 95\%: 0,03-1,98). Un solo paciente requirió internación en el Servicio de Terapia Intensiva, con adecuada evolución clínica. No se registraron óbitos.

Tabla 2. Distribución de frecuencia de los grupos farmacológicos implicados en la intoxicación, expresados en porcentajes

\begin{tabular}{lc}
\hline Tipo de fármaco & Consumo (n\%) \\
\hline Ansiolíticos & $22(30 \%)$ \\
Analgésicos & $10(14 \%)$ \\
Antihipertensivos & $2(3 \%)$ \\
Antialérgicos & $3(4 \%)$ \\
Estimulantes & $2(3 \%)$ \\
Antidepresivos & $2(3 \%)$ \\
Antibióticos & $1(1,5 \%)$ \\
Fármacos & $18(25 \%)$ \\
Desconocidos & $7(9,5 \%)$ \\
Otros & $5(7 \%)$ \\
\hline
\end{tabular}

\section{DISCUSIÓN}

El sexo femenino resultó el más implicado en la consulta por intoxicación medicamentosa, lo que coincidió con otros trabajos, que expresaron una relación mujer-hombre de 3:1.,5 En cuanto a la edad, se evidenció que la mayoría de los casos correspondían a la adolescencia tardía, que concordó con lo publicado en investigaciones previas que señalaban la población de entre 15 y 19 años como la predominante. ${ }^{6,7}$

Los psicofármacos fueron los fármacos más utilizados y, dentro de ellos, los ansiolíticos ocuparon el primer lugar. Existe uniformidad en los reportes que refieren que estos son los medicamentos más consumidos en la situación de estudio. ${ }^{4,6}$

Cabe destacar el gran porcentaje de pacientes que refirieron haber consumido los fármacos con intención suicida. En un estudio realizado en el Hospital de Ramón Y Cajal de la ciudad de Madrid, se observó que el 83,8\% de las intoxicaciones fueron voluntarias. ${ }^{8}$ Por su parte, en una investigación desarrollada entre los años 2009 y 2011 en un hospital de Turquía, la cifra fue del $92 \% .^{9}$

Las formas accidentales de consumo suelen ocurrir en la infancia, mientras que los adolescentes lo hacen de manera intencional en la mayoría de los casos. ${ }^{10}$

El dato que cobra mayor interés es el gran número de pacientes que presentan antecedentes de autoeliminación, ya sea mediante el consumo de fármacos o por otros medios. Las tasas de reintento de suicidio en adolescentes están cifradas en alrededor del 6-14\% durante el primer año de seguimiento según investigaciones previas. ${ }^{11}$ Nuestro estudio muestra cifras mayores, semejantes a estudios regionales, como es el caso del realizado en la ciudad de Córdoba en el año 2006, donde el $68,8 \%$ de los menores informaron haber presentado un evento previo. ${ }^{12}$ Por su parte, en un estudio desarrollado en la ciudad de Santiago de Chile en el año 2012, la cifra de reincidencia fue del $51 \% .^{13}$

Se ha evidenciado que la frecuencia de intoxicaciones no sigue un patrón de aumento con el paso de los años, ya que los resultados fueron variables al analizar cada uno de los períodos. Quizá esto se deba a errores en la codificación diagnóstica, que puede haber dejado en el camino casos de intoxicación medicamentosa no incluidos en el estudio.

Entre las limitantes, se mencionan el carácter descriptivo y retrospectivo de la investigación y que se trata de una serie acotada de casos. Sin embargo, teniendo en cuenta las características 
poblacionales de la ciudad de Bahía Blanca, se podría decir que el número de adolescentes que consultaron por intoxicación medicamentosa fue considerable.

Se considera que, a futuro, se deberían desarrollar trabajos prospectivos sobre esta temática y utilizar métodos cualitativos que permitan profundizar aspectos relacionados con el adolescente.

\section{CONCLUSIÓN}

En este estudio, se destaca la predominancia de mujeres en cuanto a la consulta al Servicio de Emergencias por intoxicación medicamentosa. Los ansiolíticos fueron los que se presentaron con mayor frecuencia y con un importante porcentaje de antecedentes de intoxicaciones previas.

\section{REFERENCIAS}

1. Fernández-BarocioF,Sánchez-VillegasMCS.Epidemiología de las intoxicaciones en el servicio de urgencias pediátricas de un hospital de tercer nivel. Reporte de cinco años. Archivos de Medicina de Urgenc de Méx 2013;5(1):18-24.

2. Programa Nacional de Salud Integral en la Adolescencia. Situación de la salud de las y los adolescentes en la Argentina. Buenos Aires: Ministerio de Salud - UNICEF; 2016. [Acceso: 22 de diciembre de 2017]. Disponible en: http:/ / www.msal.gob.ar/images/stories/bes/ graficos/0000000872cnt-linea-base-adolescencia-2016.pdf.
3. Galvis Pérez A, Ospina Díaz JM, Manrique Abril FG. Caracterización de la intoxicación exógena en niños y adolescentes en Sogamoso, Boyacá durante el período de 2010 a 2013. Medicas UIS 2014;27(1):9-16.

4. Dessauer B, Ortiz P, Hinostroza $\mathrm{T}$, et al. Intento de suicidio vía ingesta de fármacos en niños. Rev Chil Pediatr 2011;82(1):42-8

5. Zöhre E, Ayrik C, Bozkurt S, et al. Retrospective Analysis of Poisoning Cases Admitted to the Emergency Medicine. Arch Iran Med 2015;18(2):117-22.

6. Prada DB, Evangelista M, Piola JC. Tentativas de suicidio con tóxicos en adolescentes en Rosario, Argentina. 19901998. Brazilian Pediatric News 2002;4(3):1-11.

7. Pringle K, CauppS, Shi J, et al. Analysis of intentional drug poisonings using Ohio Poison Control Center Data, 20022014. Clin Toxicol (Phila) 2017;55(7):652-8.

8. Medina González L, Fuentes Ferrer ME, Suárez Llanos JP, Arranz Peña MI, et al. Epidemiología de las intoxicaciones medicamentosasduranteun añoen el Hospital Universitario Ramón y Cajal. Rev Clin Esp 2008;208(9):432-6.

9. Kara H, Bayir A, Degirmenci S, et al. Causes of poisoning in patients evaluated in a hospital emergency department in Konya, Turkey. J Pak Med Assoc 2014;64(9):1042-8.

10. Cuesta-Revé D. Aspectos epidemiológicos del suicidio en adolescentes. Rev Mex Pediatr 2017;84(2):72-7.

11. Poblete $R$, Morell I, Cabrera D, et al. Intoxicación medicamentosa voluntaria: un paciente, dos problemas. Rev Chil Med Intensiva 2008;23(2):85-93.

12. Bella M, Fernández R, Willington JM. Identificación de factores de riesgo en intentos de suicidio en niños y adolescentes. Rev Argent Salud Pública 2010;1(3):24-9.

13. Inzunza $C$, Navia F, Catalán $P$, et al. Conducta suicida en niños y adolescentes ingresados en un hospital general. Análisis descriptivo. Rev Med Chile 2012;140(6): 751-62.

\section{Artículos seleccionados}

A partir de este número hemos cambiado en esta sección el título de Correo de la $S A P$, que estuvo presente durante más 10 años, por el de Artículos seleccionados. Los editores entendimos que este título actual era más demostrativo de lo que realmente hacemos, que es presentarles artículos que puedan ser de interés para los pediatras.

Los siguientes resúmenes y comentarios de trabajos seleccionados se encuentran disponibles en la versión electrónica de este número.

PEDIATR PULMONOL. 2018 Jan 24

Tos tipo hábito/tic: presentación y evolución con confirmación simple

(Wright MFA, et al. Habit-tic cough: Presentation and outcome with simple reassurance)

Comentario: Dr. Mario Grenoville. Comité de Neumonología. Sociedad Argentina de Pediatría.

N ENGL J MED. 2018 Feb 1;378(5):428-438.

Antecedentes de enfermedad renal en la infancia y riesgo de enfermedad renal terminal en el adulto (Calderon-Margalit $R$, et al. History of childhood kidney disease and risk of adult end-stage renal disease)

Comentario: Dr. Luis Voyer. Hospital General de Niños Pedro de Elizalde. Ciudad de Buenos Aires.

N ENGL J MED. 2018 Apr 5;378(14):1302-1312.

Cambios en el sobrepeso desde la infancia al comienzo de la edad adulta y riesgo de diabetes tipo 2 (Bjerregaard LG, et al. Change in Overweight from childhood to early adulthood and risk of type 2 diabetes)

Comentario: Dra. Marisa Armeno. Servicio de Nutrición, Hospital de Pediatría "Prof. Dr. Juan P. Garrahan". Ciudad de Buenos Aires. 